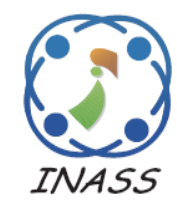

\title{
Massive MIMO: Enhancement of Spectral and Energy Efficiency for 5G Perspective
}

\author{
Prasad Rayi $^{1 *} \quad$ Makkapati Venkata Siva Prasad ${ }^{2}$ \\ ${ }^{1}$ Acharya Nagarjuna University, Guntur, \\ International School of Technology and Sciences for Women, Rajanagaram, India \\ ${ }^{2}$ Rayapati Venkata Rangarao \& Jagarlamudi Chandramouli College of Engineering, Guntur, India \\ * Corresponding author's Email: prasadrayi@gmail.com
}

\begin{abstract}
Massive Multi-Input and Multi-Output (MIMO) antenna system is considered as the key technology to improve both spectral efficiency (SE) and energy efficiency (EE) for 5G wireless systems. This paper evaluates the impact of perfect and imperfect channel state information (CSI) on the SE. The maximum SE of $200 \mathrm{bits} / \mathrm{s} / \mathrm{Hz}$ achieved in a multi-cell scenario and its limiting factors were explored. We mainly demonstrated linear precoding algorithms such as zero-forcing (ZF), maximum ratio combing (MRC), and minimum mean square error (MMSE) for SE analysis. The explicit SE and EE expressions were derived from Shannon capacity calculations for single and multi-cell scenario. The proposed ZF and MRC precoding schemes were observed with SEs of $185 \mathrm{bit} / \mathrm{s} / \mathrm{Hz}$, and $180 \mathrm{bits} / \mathrm{s} / \mathrm{Hz}$ respectively with pilot reuse factor with $\lambda=7$. The simulation results were validated by comparing the SE versus the number of base station (BS) antennas with receiving schemes by using MATLAB 2018a.
\end{abstract}

Keywords: Spectral efficiency, Energy efficiency, Multi input and multi output, Channel state information, 5G.

\section{Introduction}

Current cellular networks demand high-speed transmission with multi-user capability under interference and noise scenario. Massive multi-input and multi-output (MIMO) can improve the spectral efficiency (SE) by serving a large number of users in a cell within the same time and bandwidth. Massive MIMO is a technology with massive antennas deploying at the base station (BS) to improve SE and energy efficiency (EE) [1]. Higher data rate has achieved with prominent parameters such as a greater spectrum (Hz), larger cell density $\left(\right.$ cells $\left./ \mathrm{Km}^{2}\right)$, high $\mathrm{EE}$ and $\mathrm{SE}$ [2, 3]. The combination of a millimetre (mm)-wave communication and massive MIMO is the most promising technology for future wireless communication [4]. This combined technology can integrate and enable several schemes into one platform. Several pieces of recent research have reported $[5,6]$ to achieve high SE and EE in the fifth generation $(5 \mathrm{G})$ systems and also offer high data rate over the $4 \mathrm{G}$ systems.

The EE of the downlink (DL) mm-wave massive MIMO communication can be improved by employing hybrid-beam forming schemes [7] with reduced interference from other user terminals (UT)s. To the best of our knowledge, the achievable rate of massive MIMO mainly depends on perfect channel state information (CSI) available at the UT and the BS. However, acquiring the perfect CSI is highly limited by the coherence time and pilot contamination (PC) $[8,9]$ in large antenna systems. The impact of interference with PC is presented in [10] on the cells and UTs. It is required to obtain a maximum signal to interference plus noise ratio (SINR) to increase the SE. Since the SE strongly depends on the channel data rate. In addition to this, resource allocation also plays a key role to attain the maximum SE and EE [11 - 13].

The power allocation schemes were proposed [14] to reduce the interference both from the primary and the secondary UTs. The network 
performance notably increased when small cell access combined with Massive MIMO systems [15, 16]. The small cell access proposed $[17,18]$ in high traffic areas with more user density to increase each UT sum SE and EE. The authors of [19] addressed the adaptive spatial modulation techniques to enhance the EE with a single antenna per UT. The SE and EE of the distributed MIMO were described [20] with channel parameters such as antenna correlation, multipath fading and path loss. The degradation of the EE and SE with increasing the $\mathrm{PC}$ and RF impairment well demonstrated in [21].

Energy consumption is also the most important issue in wireless networks to achieve higher throughput. The maximization of the EE exploited [22] to mitigate the impact of the PC and channel estimation error. The EE is also maximized by the transmitting RF chain configuration [23]. The resource allocation schemes show [24] significant role in the optimization of EE with optimal antennas at the BS. The area energy efficiency (AEE) improved by a realistic power consumption model reported [25] when the number of BS antennas reaches of the infinite. The impact of the full-duplex scheme than half-duplex in massive multi-user MIMO systems with the SE reported in [26]. The issue of analytical lower bound SE in device-todevice networks well expressed [27] in a single cell. The SE also increases with a maximum ratio combining (MRC) scheme [28] in the DL massive MIMO systems with perfect and imperfect CSI. However, it was observed that the trade-off between the SE and the EE [29] is achieved by dealing with the power consumption of the transceiver into account.

Recent works on the SE of massive MIMO show that there is a maximum value for SE is achieved by serving a limited number of UTs. In this paper, we demonstrated how to achieve maximum SE and EE by serving the large number of UTs and limited number of pilot sequences. We examined the resource allocation schemes such as signal transmission and power allocation in a distributed fashion. The impact of perfect and imperfect CSI on the SE is proposed in the DL massive MIMO systems. We also addressed how many BS antennas are required to achieve maximum SE and its limiting factors were also presented. The linear precoding algorithms were demonstrated such as zero-forcing (ZF), minimum mean square error (MMSE) estimation and MRC in SE analysis. The peak value of $\mathrm{SE}$ and $\mathrm{EE}$ is achieved by deploying massive BS antennas and serving the large number of UTs. The final goal has to enhance the SE and EE with massive BS antennas in the multi-user environment.

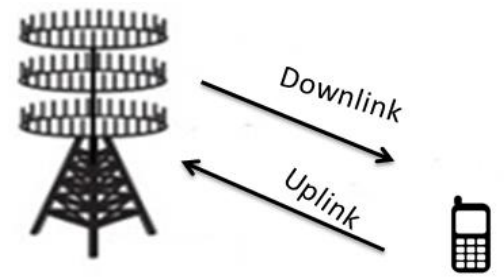

Base station

Mobile station

Fig.1 Proposed system model for massive MIMO

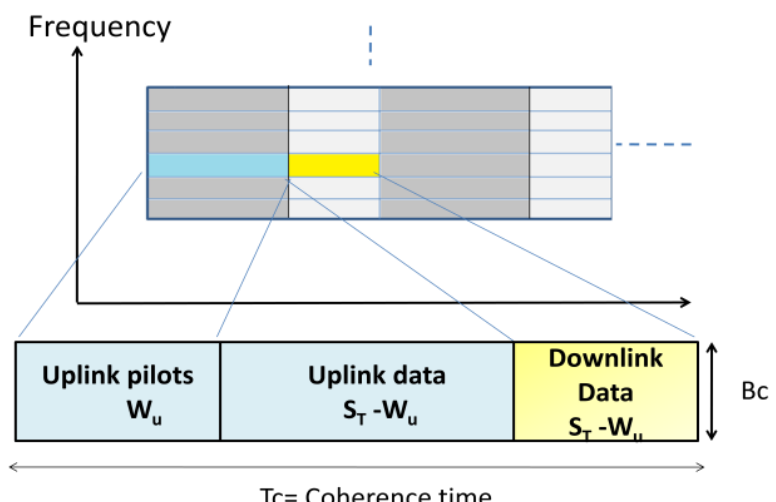

Fig.2 Proposed Frame structure for both UL and DL

Here, the most important deal is to allocate the universal resources intelligently such as time and spectrum to improve the system performance. In particular, the pilot sequences were allocated to the BSs properly to reduce the interference.

The research paper is organized as follows, the proposed system model of the massive MIMO presented in Section 2. An overview of the average SE analysis is presented with UL and DL channel estimation in section 3. The optimization of the SE and EE were presented in section. 4. The Simulation results were compared with the SE analysis and the BS antennas in section. 5. Finally, the conclusion is presented. Notation: Matrices and vectors are denoted by boldface upper and lower case symbols. Here, (.), [.] ${ }^{\mathrm{T}}$, [.] $^{+}$represent trace, transpose, and Hermitian transpose respectively. Finally, the expectation symbol is presented by E [.].

\section{Proposed system model}

In this section, we considered a proposed cellular system model shown in Fig.1. The user data are transmitted with universal frequency reuse and time. This model was implemented with $\mathrm{M}$ number of BS antennas and, $k$ single antenna UTs. The number of cells was represented by $N$. The subset of the active number of UTs is denoted by $k=$ $\left\{1,2, \ldots \ldots \ldots, K_{\max }\right\}$.

In this work, each UT has assigned to a cell such as $n \in N$ at different times and the position $Z_{n k} \in$ 
$R^{2}$. The proposed frame structure is presented in Fig.2. The universal time-frequency resources were categorically divided into frames. The number of transmitting symbols per frame was denoted by $T_{s}=$ $T_{c} B_{c}$. Here, each frame was presented with $T_{c}$ Seconds and $B_{c} \mathrm{~Hz}$. Similarly, $B_{c}$ is smaller than the coherence bandwidth of all UTs. Where $S_{T}$ denotes a coherence block length and $W_{u}$ orthogonal pilot symbols. Where $T_{c}$ is the coherence time of the channel and inverse of the coherence bandwidth. Hence, all the channel coefficients were assumed to be constant over $T_{c}$. The channel response between UT $k$ and BS $i$ in a cell $n \in N$ under non-line-ofsight propagation is given by

$$
h_{n l k} \cong C N\left(0, d_{n}(Z), Z_{i k}\right) I_{M}
$$

Where $h_{n l k} \in C^{N}$ is distributed as a zero-mean circularly symmetric complex Gaussian (ZMCSCG) process. Where $I_{M}$ denotes the identity matrix with size $\mathrm{M} \times \mathrm{M}$. Where $d_{n}(z)$ denotes the deterministic function and also provides channel variation. The received CSI in the uplink (UL) channel estimation is also used for the DL by using channel reciprocity. The remaining $S_{T}-W_{u}$ numbers of symbol resources were utilized for both the UL and DL.

\subsection{Uplink channel estimation}

In this section, we considered the UL received signal at the BS $i$ from the UT: $U_{i k} \in C^{M}$. It is represented as follows,

$$
U_{i k}=\sum_{n=1}^{N} \sum_{k=1}^{K} h_{n i k} S_{n k}+n_{i k}
$$

Here, $S_{n k}$ is the symbol transmitted from UT $k$ to the cell $n$. The normalization of the signal is denoted by $E\left[S_{n k}\right]^{2}$. The transmitted power correspondingly to the UL is expressed as $\rho_{n k} \geq 0$. The noise term $n_{i k} \in C^{M}$ is modelled as the additive white Gaussian distributed process with zero mean and variance equal to unity. The UL power assumed as a fixed value in the channel model [30].

We considered the stochastic aware power control (SAPC) scheme. This channel model requires CSI from all users in a cell by using a rapid feedback mechanism. The transmitted power from the cell $n$ to the UT $k$ is given by

$$
p_{n k}=\frac{\rho}{d_{n}\left(Q_{n k}\right)}
$$

Where $\rho$ denotes the design parameter, and $Q_{n k}$ undesired signal. The SAPC supports both the EE and uniform end-user experience. To do this, analog-to-digital converter should be designed to minimize the effect of near-far blockage [31] with the lower bound achievable rate.

\section{Average Spectral efficiency}

In this section, we proposed the average SE with a pilot based channel estimation for the UL and DL massive MIMO systems. The proposed system model implemented with the BS transmit the data with precoding vector in the DL. The signals are coherently combined and coordinated at the receiving antennas. However, this process requires the perfect CSI at the BS $i$ from the UL channel [32-33]. To improve the capacity of massive MIMO systems, spectral resources were reused in different cells. The pilot signals used in one cell affect the pilot signals in another cell. Since the PC limits the achievable data rate and transmitted power at the BS. The transmitted pilot signals are represented by a fixed codebook given by

Where

$$
\vartheta=\left\{u_{1}, u_{2}, \ldots, u_{n}\right\}
$$

$$
u_{a 1}^{H} u_{a 2}= \begin{cases}w & a_{1}=a_{2} \\ 0 & a_{1} \neq a_{2}\end{cases}
$$

Where $\vartheta$ denotes the index used in the pilot book. It is termed as a reference signal and also known to the UT $k$ and the BS in a cell $n$. The Eq. (4) provides the UL channel estimation with the transmission of the pilot tones over the symbols $W_{u}$. The UL received signal at the BS $i$ is presented as

$$
Y_{i}=\sum_{n \in N} \sum_{k=1}^{K} \sqrt{\rho} h_{i n k} z_{j i k}^{T}+n_{i k}
$$

The BS transmits data and uses the dominance of the MRC linear precoding in the DL. The UL effective channel is represented by the MMSE estimate [34] as follows,

$$
\hat{h}_{i n k}=D_{i, n} Y_{i}\left(\varnothing_{i}^{T}\right)^{-1} a_{j n k}
$$

Where $\quad D_{i, n}=\frac{d_{i}\left(Q_{n k}\right)}{d_{n}\left(Q_{n k}\right)}$

Where $D_{i, n}$ defines the relation between the desired signal and an interference signal from the BS $i$ to the UT $k$ in a cell $n$. The ratio is equal to unity for any cell. Where $a_{j n k}$ represents $j^{\text {th }}$ column of the matrix $I_{w}$, which is due to the impact of the PC. The fundamental difference between the MMSE and conventional massive MIMO estimators are explained by using two factors. First one, the pilot allocation is provided by the MMSE estimator. 
The second one, the channel estimation error modelled by the covariance matrix $C_{i n k} \in C^{M \times M}$ as follows,

$$
\begin{aligned}
C_{i n k} & =E\left[h_{i n k}-\hat{h}_{i n k}\right]\left[h_{i n k}-\hat{h}_{i n k}\right]^{H} \\
& =\rho D_{i, n}\left[1-\frac{D_{i, n} W_{u}}{\emptyset_{i}}\right] I_{M}
\end{aligned}
$$

Where the received covariance matrix $\emptyset_{i} \in$ $C^{w_{u} \times w_{u}}$ is given by

$$
\emptyset_{i}=\sum_{n \in N} \sum_{l=1}^{k} D_{i l} Z_{j n l} Z_{j n l}^{H}+\frac{\sigma^{2}}{\rho} I_{w}
$$

Here, $I_{w}$ denotes the identity matrix. The estimated mean square error (MSE) is derived as $M S E_{\text {ink }}=\operatorname{tr}\left(C_{\text {ink }}\right)$. finally, each BS selects only $W_{u}$ orthogonal pilot signals and resolves the pilots into spatial dimensions. The channel estimate is given by

$$
H_{\vartheta, i}=Y\left(\phi_{i}^{T}\right)^{-1} U_{\vartheta}
$$

$$
\text { Where } U_{\vartheta}=\left[u_{1}^{*}, u_{2}^{*}, \ldots \ldots, u_{w}^{*}\right]
$$

\subsection{Uplink achievable spectral efficiency}

In this work, the sum SE of network transmissions in a cell is measured as the number of bits transmitted per second per Hertz (bit/s/Hz). Inter-cell interference (ICI) limits the performance of the massive MIMO systems. An interference alignment technique was employed [35] to decrease the ICI at the BS with the Poisson point process. The performance of the massive MIMO system highly depends on the channel estimation and receive combing scheme. In order to decrease the interference, the combining technique could be addressed in the form of either active or passive. Thus, the MRC scheme with passive interference is given by

$$
t_{M R C}=H_{\vartheta, i} a_{j i k} \hat{h}_{i i k}
$$

Where $H_{\vartheta, i}$ is the channel matrix and $\hat{h}_{i i k}$ effective channel estimate with MRC. The active interference rejection schemes substantially attain more gain over the passive interference rejection schemes. Since the receive combining is considered the channels as orthogonal. The impact of pilot allocation and interference on the SE from other cells is presented as follows:

$$
S E^{U L}=\rho\left[1-\frac{W_{u}}{S_{T}}\right] E\left[\log _{2}\left(1+S I N R_{i k}\right)\right]
$$

The passive interference rejection scheme $\mathrm{ZF}$ was given as follows:

$$
t_{Z F}=H V_{i} E\left[V_{i}^{H} H_{\vartheta, i}^{H} \widehat{H}_{\vartheta, i} V_{i}\right]^{-1} a_{j i k}
$$

Where $V_{i}=\left[a_{1 i k}, \ldots \ldots, a_{j i k}\right] \in C^{W \times K}$

The ergodic data rate with the lower bound approximation is given by

$$
S E_{i}^{U L}=k \zeta^{u l}\left(1-\frac{W_{u}}{S_{T}}\right) \log _{2}\left(1+\frac{1}{I_{i}}\right)
$$

Where $I_{i}$ denotes the interference

$$
I_{i}=\sum\left(\alpha_{i n}+\frac{\alpha_{i n}-\alpha}{T_{s c h}}\right)+\frac{\left(\sum \alpha_{i n} G_{i n}^{s c h}+\frac{\sigma}{\rho}\right)\left(\sum \alpha_{i n} G_{i n}^{s c h}+\frac{\sigma}{\mu}\right)}{G_{s c h}}
$$

Where $\alpha_{i n}=E\left[D_{i, n}^{w}\right]$ denotes the propagation parameter and $G_{i n}^{s c h}$ provides the combing scheme at the receiver. The interference in Eq. (15) highly depends on the $G_{i n}^{s c h}$. The ZF scheme is designed as follows,

$$
\begin{aligned}
T_{Z F}= & \left\{K\left(1-\frac{\alpha_{\text {in }}}{\sum_{N_{i}(\mu)} \alpha_{i n}+\frac{\sigma^{2}}{\mu_{p}}}\right)\right\} \text { if } n \in N_{i}(\mu) \\
& =K \quad \text { if } n \notin N_{i}(\mu)
\end{aligned}
$$

The second part of the numerator in Eq. (16) represents the noise power at the receiving scheme.

\subsection{Downlink achievable spectral efficiency}

We considered the DL achievable SE by deriving the channel estimation with precoded data at the BS. It is identical to the UL channel estimation. The DL received signals were combined coherently from the $\mathrm{M}$ channel inputs. Here, the desired signals were selected properly from the desired UTs by suppressing undesired signals from the undesired UTs. The precoded vector is presented as follows,

$$
W_{i k}=\sqrt{\frac{P_{i k}}{E_{\{h\}}\left\{\left|g_{i k}\right|^{2}\right\}}} g_{i k}^{*}
$$


Where $P_{i k}$ is transmitting power from the BS $i$ to the UT $k$. The ZF suppresses the ICI by properly selecting the $g_{i k}$. The DL SE with the precoded vector and transmitting power is given by

$$
S E^{D L}=\zeta\left(1-\frac{W_{i k}}{S_{T}}\right) E_{\{Q\}} \log _{2}\left(1+\operatorname{SIN} R_{i k}\right)
$$

Where

$$
\operatorname{SINR}_{n k}=\frac{P_{i k}\left\{E_{\{h\}}\left\{g_{i k}^{H}\left|h_{i i k}\right|^{2}\right\}\right.}{\left\{E_{\{h\}}\left|g_{c m}\right|^{2}\right\}-\frac{\left.P_{i k}\left\{E_{\{h\}} g_{i k}^{H}\left|g_{i k}^{H} h_{i k}\right|^{2}\right\}\right\}}{\left\{E_{\{h\}}\left|g_{i k}^{H}\right|^{2}\right\}}+\sigma^{2}}
$$

Where $\zeta$ is a path loss exponent. Traditionally, the BS allocates power to the different UTs based on the UL CSI in the precoding. Finally, the MMSE eliminates both the inter-user interference and ICI by selecting $g_{i k}$. The normalization of spatial directivity provides analytical tractability of the channel as follows,

$$
S E=k\left(1-\frac{W_{i k}}{S_{T}}\right) \log _{2}\left(1+\frac{1}{I_{i}}\right)
$$

Here, the Eq. (20) is also similar to the UL achievable SE. Since it is designed based on the knowledge of the UL received CSI. The MRC scheme amplifies the desired signal and eliminates inter-user interference.

\section{Optimization of Spectral and Energy efficiency}

\subsection{Single and multi-cell massive-MIMO system}

In this section, we presented the SE on a single and multi-cell massive MIMO system with perfect and imperfect CSI available at the BS. It can be presented as follows:

$$
\left.\begin{array}{c}
R_{p e r}^{S}=\sum_{k=1}^{K} R_{p e r, k}^{S} \\
R_{\text {imp }}^{S}=\frac{T_{c}-\tau}{T_{c}} \sum_{k=1}^{K} R_{\text {imp }, k}^{S}
\end{array}\right\}
$$

Where $S \in\{Z F, M R C, M M S E\}$, corresponds to receive combining schemes in the Massive MIMO system. The EE is represented with the SE for perfect and imperfect CSI as follows:

$$
\left.\begin{array}{c}
\eta_{\text {imp }}^{S}=\frac{R_{\text {imp }}^{S}}{P_{u}} \\
\eta_{p e r}^{S}=\frac{R_{p e r}^{S}}{P_{u}}
\end{array}\right\}
$$

Where $\eta_{\text {per }}^{S}$ denotes the EE of receiving scheme with perfect CSI, and $\eta_{i m p}^{S}$ EE for imperfect CSI. The fundamental cellular concept has been proposed in [36] with hexagonal cellular geometry. The impact of co-channel interference on the frequency reuse pattern with hexagonal coverage was highlighted in [37]. We considered the cellular network with the pilot reuse pattern $\lambda=7$.

\subsection{Maximum ratio combining receiver}

We considered the MRC scheme for the SE and EE of the massive MIMO with perfect and imperfect CSI are presented as follows,

$$
\begin{aligned}
& R_{i m p}^{S}= \frac{T_{c}-\tau}{T_{c}} K \log _{2}\left(1+\frac{\tau(M-1) P_{u}}{\tau(K-1) P_{u}^{2}+(K+\tau) P_{u}+1}\right) \\
& \eta_{i m p}^{M R C}=\frac{R_{i m p}^{M R C}}{P_{u}} \\
& \lim _{P_{u} \rightarrow 0} \eta_{i m p}^{M R C}=\lim _{P_{u} \rightarrow 0} \frac{1}{P_{u}} R_{i m p}^{M R C}=0 \\
& \lim _{P_{u} \rightarrow \infty} \eta_{i m p}^{M R C}=\lim _{P_{u} \rightarrow \infty} \frac{1}{P_{u}} R_{i m p}^{M R C}
\end{aligned}
$$

The SE is proportional and also increasing function of power $P_{u}$ from Eq. (23). Hence the SE increases with the $\mathrm{EE}$ under the low power regime. Therefore, the power $P_{u} \ll 1$ shows the low power regime and approximated as $P_{u}^{2}$ to achieve the maximal SE When $d>0$. The fundamental relationship between the $\mathrm{SE}$ and $\mathrm{EE}$ is presented for the MRC scheme with $P_{u} \ll 1$ given by

$$
\eta_{i m p}^{M R C}=\sqrt{\frac{T_{c}-\tau}{T_{c}} K \log _{2}(e) \tau(M-1) R_{i m p}^{M R C}}
$$

Here, Eq. (27) is approximated from the Taylor series expansion $P_{u} \ll 1$.

\subsection{Zero forcing receiver}

Here, the ZF analysis is similar to the MRC scheme. The main drawback of the ZF receiver is the noise amplification. The SE and EE expressions with $\mathrm{ZF}$ scheme are written as follows:

$$
\begin{gathered}
R_{i m p}^{Z F}=\frac{T_{c}-\tau}{T_{c}} k \log _{2}\left(1+\frac{\tau(M-k) P_{u}^{2}}{(k+\tau) P_{u}+1}\right) \\
\eta_{i m p}^{Z F}=\frac{1}{P_{u}} R_{i m p}^{Z F}
\end{gathered}
$$


The achievable SE with ZF scheme is also increasing function of $P_{u}$. The $\mathrm{SE}$ and the EE were derived with Taylor series expansion as follows,

$$
\begin{gathered}
R_{i m p}=\frac{T_{c}-\tau}{T_{c}} k \log _{2}(e) \tau(M-1) R_{i m p}^{M R C} P_{u}^{2} \\
\eta_{i m p}^{Z F}=\sqrt{\frac{T_{c}-\tau}{T_{c}} k \log _{2}(e) \tau(M-1) R_{i m p}^{Z F}}
\end{gathered}
$$

\section{Simulation results and discussion}

In this section, the simulation results were presented and compared for the linear processing algorithms. We demonstrated the impact of perfect and imperfect CSI on the SE with increasing the BS antennas. The transmitted power per user $P_{u}=$ $10 \mathrm{~dB}$ for the UL channel estimation. The low optimum coherence block length is equal to $S_{T}=$ $200(200 \mathrm{KHz}$ for coherence bandwidth and $1 \mathrm{~ms}$ for coherence time) and path loss exponent $\gamma=3.7$. The payload data are encoded with 5 bits/symbol. The simulation results were demonstrated by using the modulation scheme 64- quadrature amplitude modulation (QAM) with $3 / 4$ code rate. The simulation parameters were presented in Table 1 and performed by using MATLAB 2018a.

It was noticed that the improving the value of $S_{T}$ greater than 400 attains low system performance. Specifically, when the BS antennas of the system were observed by $M=100$. Since the $M / K$ ratio is very small. Hence, the simulation results show that increasing the BS antennas more than $\mathrm{M}=500$, can increase the SE in a cell. Since the increasing coherence block length could schedule a large number of UTs in a system. But ICI is the most significant problem which limits the SE. The impact of imperfect CSI on the SE was demonstrated in [38-40], when the BS antennas were $M=300$. By considering MMSE scheme the ICI can be decreased with increasing antennas at the BS.

Fig. 3 shows the variation of per-UT SE versus antennas at the BS with perfect and imperfect CSI. The peak value of per-UT SE of $2.7 \mathrm{bits} / \mathrm{s} / \mathrm{Hz}$ is achieved with MMSE when the BS antennas were $M=340$ with perfect CSI. It is noticed that ZF scheme is achieved the per-UT SE of $2.5 \mathrm{bits} / \mathrm{s} / \mathrm{Hz}$ with $M=270$ and imperfect CSI respectively. Finally, the low per-UT of $1 \mathrm{bits} / \mathrm{s} / \mathrm{Hz}$ is observed with MRC $\mathrm{M}=60$ and imperfect CSI. It is identified that the per-UT SE reached to a peak value with maximum BS antennas by serving large numbers of UTs. The per-UT SE is saturated to a constant value due to the impact of imperfect CSI.
Table 1. Simulation parameters

\begin{tabular}{|l|l|l|}
\hline S.No & \multicolumn{1}{|c|}{ Parameter } & \multicolumn{1}{c|}{ Value } \\
\hline 1 & Coherence block length & 200 \\
\hline 2 & Path-loss exponent & 3.7 \\
\hline 3 & Pilot reuse pattern & 7 \\
\hline 4 & SNR & $-10: 1: 25$ \\
& & $d B$ \\
\hline 5 & Maximum number of UTs & 100 \\
\hline
\end{tabular}

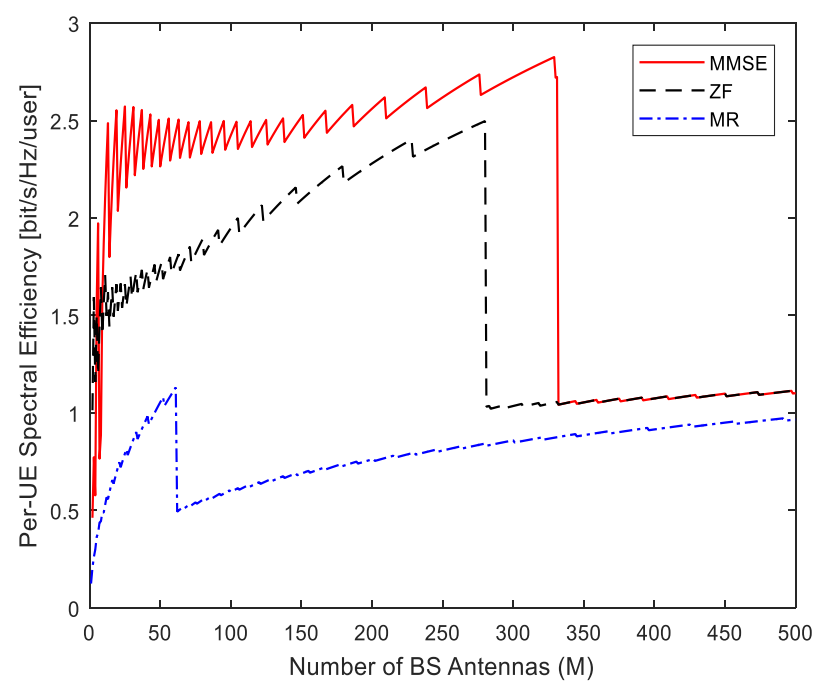

Fig.3 per-UT SE Vs number of BS antennas with $M=500$

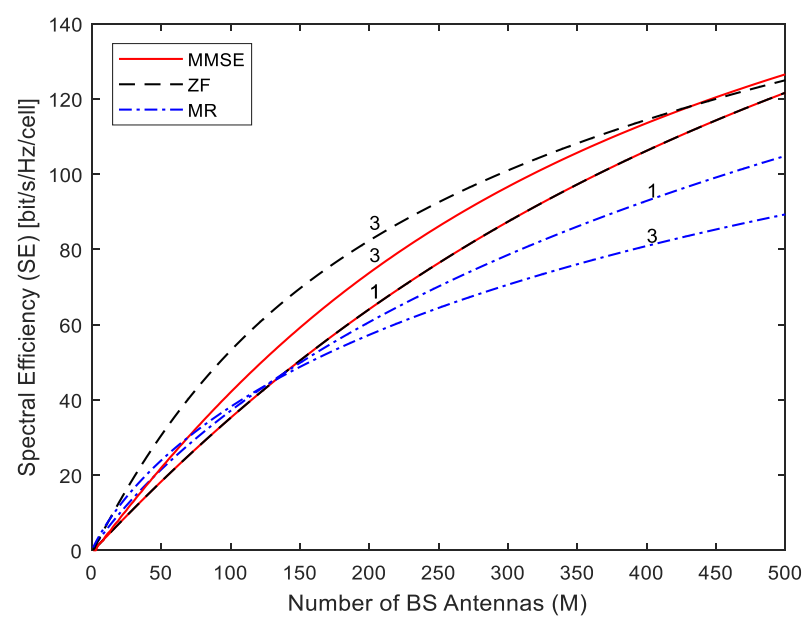

Fig.4 SE Vs antennas at the BS with $M=500$

In this section, simulation results were presented in real-time by considering the perfect and imperfect CSI. The Fig. 4 shows the variation of SE versus the BS antennas with imperfect CSI. In this case, the maximum number of antennas is equal to $\mathrm{M}=500$. We observed that the SE of the massive MIMO system increases linearly with antennas at the BS. The maximum SE $125 \mathrm{bit} / \mathrm{s} / \mathrm{Hz}$ was observed by using MMSE with $\mathrm{M}=500$ and serving 20 UTs. Similarly, the ZF scheme was achieved $120 \mathrm{bit} / \mathrm{s} / \mathrm{Hz}$ 
with imperfect CSI and 64-QAM. Finally, MRC was noticed as $110 \mathrm{bits} / \mathrm{s} / \mathrm{Hz}$ only. Interestingly, the simulation results show remarkable improvement of 25 bits/s/Hz with MMSE compared to the MRC respectively in the DL scenario. The maximum SE is decreased by $5 \mathrm{bits} / \mathrm{s} / \mathrm{Hz}$ with $\mathrm{ZF}$ compared to the MMSE with $M=500$.Since the impact of interference from Eq. (15) due to imperfect CSI in the DL scenario.

Fig. 5 shows the variation of SE with the UTs and perfect CSI. We considered the number of UTs were 100 . We identified that the SE exponentially increases with UTs. The peak value of SE 80 $\mathrm{bit} / \mathrm{s} / \mathrm{Hz}$ is observed with MMSE for 25 UTs. We noticed that by increasing the $\mathrm{BS}$ antennas the $\mathrm{ZF}$ will follow the MMSE scheme. Where the MRC scheme is achieved only $60 \mathrm{bits} / \mathrm{s} / \mathrm{Hz}$ due to the impact of imperfect CSI. Whilst changing the pilot reuse factor has an immense impact on the SE expressions Eq. (14), Eq. (18) presented in the section. 3. In order to achieve the high SE, the number of BS antennas as $M=2000$, and the pilot reuse factor $\lambda=7$. Thus the pilot signal consumes 2 to $40 \%$ of the total frame in the simulation.

Similarly, Fig.6 illustrates the variation of the $\mathrm{SE}$ when the maximum number of the BS antennas is equal to $\mathrm{M}=2000$. By increasing the $\mathrm{BS}$ antennas to 2000, the large variation was observed in SE by serving 100 UTs. From the simulation results, it has been identified that the SE of the massive MIMO system saturates when the BS antennas were $M=2000$. Table 2 shows comparison of SEs for the DL scenario with perfect and imperfect CSI by using the $\mathrm{BS}$ antennas $\mathrm{M}=2000$. Here, the peak value of SE was observed as $200 \mathrm{bits} / \mathrm{s} / \mathrm{Hz}$ by using the BS antennas $\mathrm{M}=2000$ with proposed MMSE and perfect CSI respectively. But it was noticed only $11 \mathrm{bit} / \mathrm{s} / \mathrm{Hz}$ from the results [1], and $170 \mathrm{bits} / \mathrm{s} / \mathrm{Hz}$ [30] due to the impact of imperfect CSI. The peak SE of 180 bits/s/Hz [41] was obtained by using MMSE with the $\mathrm{BS}$ antennas $\mathrm{M}=1500$. It is observed that there is an improvement of $20 \mathrm{bits} / \mathrm{s} / \mathrm{Hz}$ in the proposed MMSE with the results of [41], $30 \mathrm{bits} / \mathrm{s} / \mathrm{Hz}$ [30], and $189 \mathrm{bits} / \mathrm{s} / \mathrm{Hz}$ [1] with perfect CSI scenario.

Similarly, the proposed ZF scheme is obtained the SE 185 bits/s/Hz. But, it was observed only 8 bits/s/Hz [1], $165 \mathrm{bits} / \mathrm{s} / \mathrm{Hz}$ [30], and $170 \mathrm{bits} / \mathrm{s} / \mathrm{Hz}$ [41] with ZF and imperfect CSI. Finally, the proposed MRC is noticed with $180 \mathrm{bits} / \mathrm{s} / \mathrm{Hz}$ with perfect CSI and outperformed than the result of [1] with $3 \mathrm{bits} / \mathrm{s} / \mathrm{Hz}, 150 \mathrm{bits} / \mathrm{s} / \mathrm{Hz}$ [30], and 160 bits/s/Hz [41] respectively.

In this work, it was identified that there was an enhancement of $15 \mathrm{bits} / \mathrm{s} / \mathrm{Hz}$, and $20 \mathrm{bits} / \mathrm{s} / \mathrm{Hz}$

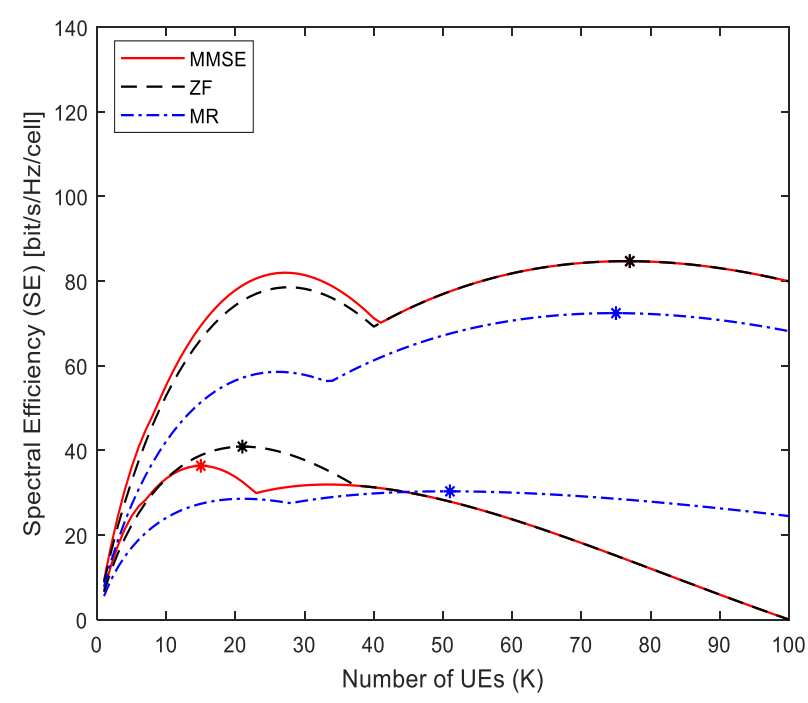

Fig.5 SE Vs number of UTs with $K=100$

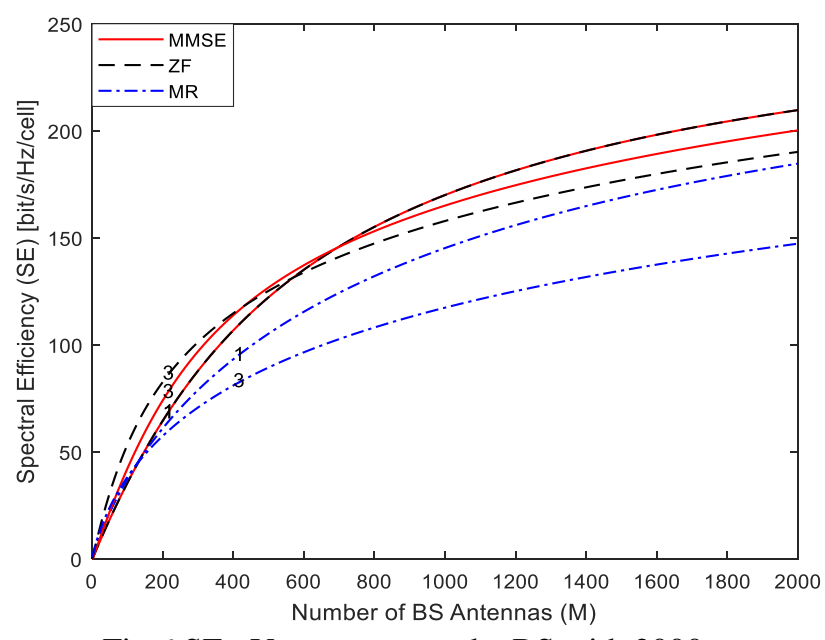

Fig.6 SE Vs antennas at the BS with 2000.

Table 2. Comparison of SEs with linear schemes

\begin{tabular}{|c|c|c|}
\hline $\begin{array}{c}\text { Precoding } \\
\text { scheme }\end{array}$ & SE (bits/s/Hz) & $\begin{array}{c}\text { Proposed SE } \\
\text { (bits/s/Hz) }\end{array}$ \\
\hline MRC & $\begin{array}{c}3[1], \\
150[30], \\
160[41]\end{array}$ & 180 \\
\hline ZF & $\begin{array}{c}8[1], \\
165[30], \\
170[41]\end{array}$ & 185 \\
\hline & $11[1]$, & \\
MMSE & $170[30]$, \\
& $180[41]$ & 200 \\
\hline
\end{tabular}

associated with $\mathrm{ZF}$ and MRC when compared to [41]. The SEs were increased with proposed $\mathrm{ZF}$ and $\mathrm{MRC}$ by $20 \mathrm{bits} / \mathrm{s} / \mathrm{Hz}$, and $30 \mathrm{bits} / \mathrm{s} / \mathrm{Hz}$ when compared with results of [30]. The improvement of SE $177 \mathrm{bits} / \mathrm{s} / \mathrm{Hz}$ is observed with the proposed $\mathrm{ZF}$ and MRC when compared with the results of [1]. Our simulation results outperformed than the results of $[1,30,41]$ when the BS antennas $M=2000$. The SE is decreased by 20 bits $/ \mathrm{s} / \mathrm{Hz}$ and $30 \mathrm{bits} / \mathrm{s} / \mathrm{Hz}$ 
[41] from the proposed ZF and MRC during the DL transmission. This can be demonstrated due to the impact of the interference in Eq. (15). Here, the interference was introduced due to imperfect CSI in the UL channel estimation.

The simulation results validate the peak SE is achieved by using the MMSE and ZF with perfect CSI. It was identified that low SE was observed with MRC and remaining scenarios due to imperfect CSI and degradation of antennas at the BS. From the results observed in this section and in section. 4, the maximum SE is achieved with antennas at the BS varying by using linear precoding algorithms. The simulation results also validate that the $\mathrm{ZF}$, MMSE and MRC with high hardware quality were considered to improve the SE in massive MIMO applications.

\section{Conclusion}

In this paper, the maximum SE of $200 \mathrm{bits} / \mathrm{s} / \mathrm{Hz}$ achieved for the multi-cell system and its limiting factors were explored. We have investigated the optimization of achievable SE and EE with perfect and imperfect CSI in massive MIMO systems. The process of estimating the channel required $40-50 \%$ of coherence blocks and the remaining blocks used for the training purpose. We derived the exact expressions for upper and lower SE scenario. The simulation results were in good agreement with theoretical Shannon capacity calculations. The SE results obtained based on the parameters, such as varying antennas at the BS M, optimal UTs $k$, and the pilot reuse factor $\lambda=7$. It has been observed that the ZF provide better SE than the MRC under best and worst case interference regime. It was noticed that the MMSE method achieved the highest SE of $200 \mathrm{bit} / \mathrm{s} / \mathrm{Hz}$ over the other reported schemes with limited UTs. But the MRC scheme was obtained with lower SE 180 bits/s/Hz with more UTs. The ZF approached the MMSE under the best case interference with SE of $185 \mathrm{bit} / \mathrm{s} / \mathrm{Hz}$. It was shown that the MMSE outperformed than the ZF under the worst case scenario. It was identified that the BS antennas increase to a larger value when the SE advanced to a constant value with perfect CSI. But the SE drastically decreased to a lower value with imperfect CSI.

Furthermore, it was validated experimentally that the imperfect CSI has a larger impact on the SE. The multiplexing techniques, ZF, MMSE and MRC with high hardware quality could be considered to improve the SE in future massive MIMO applications.

\section{References}

[1] H. Q. Ngo, E. G. Larsson, and T. L. Marzetta, "Energy and Spectral Efficiency of Very Large Multiuser MIMO Systems", IEEE Transactions on Communications, Vol.61, No.4, pp.14361449, 2013.

[2] L. Lu, G.Y. Li, A.L. Swindlehurst, A. Ashikhmin, and R. Zhang, "An Overview of Massive MIMO: Benefits and Challenges", IEEE Journal of Selected Topics in Signal Processing, Vol. 8, No. 5, pp.742-758, 2014.

[3] T.L. Marzetta, "Noncooperative Cellular Wireless with Unlimited Numbers of Base Station Antennas", IEEE Transactions on Wireless Communications, Vol.9, No.11, pp.3590-3600, 2010.

[4] A. L. Swindlehurst, E. Ayanoglu, P. Heydari, and F. Capolino, "Millimeter-wave massive MIMO: the next wireless revolution?", IEEE Communications Magazine, Vol. 52, No. 9, pp. 56-62, 2014.

[5] J.G. Andrews, S. Buzzi, W. Choi, S. V. Hanly, A. Lozano, A. C. K. Soong, and J. C. Zhang, "What Will 5G Be?", IEEE Journal on Selected Areas in Communications, Vol. 32, No. 6, pp. 10651082, 2014.

[6] A. Osseiran, F. Boccardi, V. Braun, K. Kusume, P. Marsch, M. Maternia, O. Queseth, M. Schellmann, H. Schotten, H. Taoka, H. Tullberg, M. A. Uusitalo, B. Timus, and M. Fallgren, "Scenarios for 5G mobile and wireless communications: the vision of the METIS project", IEEE Communications Magazine, Vol. 52, No. 5, pp. 26-35, 2014.

[7] J. Jing, C. Xiaoxue, and X. Yongbin, "Energyefficiency based DL multi-user hybrid beamforming for millimeter wave massive MIMO system", The Journal of China Universities of Posts and Telecommunications, Vol. 23, No. 4, pp. 53-62, 2016.

[8] U. Amin, S. Anwar, W. Khan, and S. I. Khan, "Energy efficiency analysis of single-cell massive MIMO under pilot contamination", In: Proc. of International Conf. on Applied Sciences and Technology, pp.704-709, 2016.

[9] J. Chen, H. Chen, H. Zhang, and F. Zhao, "Spectral-Energy Efficiency Tradeoff in RelayAided Massive MIMO Cellular Networks With Pilot Contamination", IEEE Access, Vol. 4, pp. 5234-5242, 2016.

[10] J. Liu, Y. Li, H. Zhang, S. Guo, and D. Yuan, "Pilot Contamination Precoding Assisted Sum Rate Maximization for Multi-cell Massive 
MIMO Systems", In: Proc. of International ITG Workshop on Smart Antennas, pp. 1-6, 2016.

[11] Y. Li, C. Tao, L. Liu, and L. Zhang, "Optimal Resource Allocation for Massive MIMO over Spatially Correlated Fading Channels", In: Proc. of IEEE Conf. on Vehicular Technology, pp.1-5, 2016.

[12] Y. Wang, C. Li, Y. Huang, D. Wang, T. Ban, and L. Yang, "Energy-Efficient Optimization for DL Massive MIMO FDD Systems With Transmit-Side Channel Correlation", IEEE Transactions on Vehicular Technology, Vol. 65, No. 9, pp. 7228-7243, 2016.

[13] Y. Wang, W. Song, C. Li, Y. Huang, S. Li, and L. Yang, "Optimal Energy-Efficient Resource Allocation for Massive MIMO FDD DL System", In: Proc. of IEEE Conf. on Vehicular Technology, pp.1-5, 2015.

[14] H. Tran, G. Kaddoum, and F. Gagnon, "Power allocation for cognitive underlay networks with spectrum band selection", Physical Communication, Vol. 21, pp. 41-48, 2016.

[15] V. Jungnickel, K. Manolakis, W. Zirwas, B. Panzner, V.Braun, M. Lossow, M. Sternad, R. Apelfrojd, and T. Svensson, "The role of small cells, coordinated multipoint, and massive MIMO in 5G", IEEE Communications Magazine, Vol.52, No.5, pp.44-51, 2014.

[16] J. Hoydis, K. Hosseini, S. Ten Brink, and M. Debbah, "Making smart use of excess antennas: Massive MIMO, small cells, and TDD", Bell Labs Technical Journal, Vol. 18, No. 2, pp. 521, 2013.

[17] H. D. Nguyen and S. Sun, "Massive MIMO versus small-cell systems: Spectral and energy efficiency comparison", In: Proc. of IEEE International Conf. on Communications, pp.1-6, 2016.

[18] P. Rayi and M.V.S. Prasad, "Optimization of energy and spectral efficiency of massive MIMO-small cell system", In: Proc. of International Conf. on Smart Technologies and Management for Computing, Communication, Controls, Energy and Materials, pp.233-238, 2015.

[19] P. Patcharamaneepakorn, S. Wu, C. X. Wang, H. Aggoune, M. Alwakeel, X. Ge, and M. Di Renzo, "Spectral, Energy and Economic Efficiency of 5G Multi-cell Massive MIMO Systems with Generalized Spatial Modulation", IEEE Transactions on Vehicular Technology, Vol.65, No.12, pp.9715-9731, 2016.

[20] G. N. Kamga, M. Xia, and S. Aïssa, "SpectralEfficiency Analysis of Massive MIMO Systems in Centralized and Distributed Schemes", IEEE
Transactions on Communications, Vol. 64, No.5, pp.1930-1941, 2016.

[21] X. Fang, Y. Zhang, H. Cao, and N. Ying, "Spectral and energy efficiency analysis with massive MIMO systems", In: Proc. of IEEE International Conf. on Communication Technology, pp.837-843, 2015.

[22] W. Xu, S. Li, S. Wang, Z. Feng, J. Lin, and A. Vasilakos, "Joint Parameter Selection for Massive MIMO: An Energy-Efficient Perspective", IEEE Access, Vol.4, pp.3719-3731, 2016.

[23] T. H. Tai, W. H. Chung, and T. S. Lee, "A Low Complexity Antenna Selection Algorithm for Energy Efficiency in Massive MIMO Systems", In: Proc. of IEEE International Conf. on Data Science and Data Intensive Systems, pp.284-289, 2015.

[24] M.A. Abuibaid and S.A. Çolak, "EnergyEfficient Massive MIMO System: Exploiting User Location Distribution Variation", International Journal of Electronics and Communications, Vol. 72, pp.17-25, 2017.

[25] Y. Xin, D. Wang, and X. You, "Area spectral efficiency and area energy efficiency analysis in massive MIMO systems", In: Proc. of International Conf. on Wireless Communications \& Signal Processing, pp.1-5, 2015.

[26] P. Xing, J. Liu, and C. Zhai, "Spectral Efficiency of the Uplink Channel in the SharedAntenna Full-Duplex Massive MU-MIMO System", In: Proc. of IEEE International Conf. on Vehicular Technology, pp.1-5, 2016.

[27] X. Lin, R. W. Heath, and J. G. Andrews, "Spectral efficiency of massive MIMO systems with D2D underlay", In: Proc. of IEEE International Conf. on Communications, pp. 4345-4350, 2015.

[28] Y. Lin, X. Li, W. Fu, and Y. Hei, "Spectral efficiency analysis for DL massive MIMO systems with MRT precoding", China Communications, Vol.12, pp.67-73, 2015.

[29] S. Mukherjee and S. K. Mohammed, "Energyspectral efficiency trade-off for a massive SUMIMO system with transceiver power consumption", In: Proc. of IEEE International Conf. on Communications, pp.1938-1944, 2015.

[30] E. Björnson, E. G. Larsson, and M. Debbah, "Massive MIMO for Maximal Spectral Efficiency: How Many Users and Pilots Should Be Allocated?", IEEE Transactions on Wireless Communications, Vol.15, No.2, pp.1293-1308, 2016. 
[31] Y. Li, C. Tao, L. Liu, G. Seco-Granados, and A. L. Swindlehurst, "Channel estimation and uplink achievable rates in one-bit massive MIMO systems", In: Proc. of IEEE Workshop on Sensor Array and Multichannel Signal Processing, pp.1-5, 2016.

[32] Y. Li, C. Tao, G. Seco-Granados, A. Mezghani, A. L. Swindlehurst, and L. Liu, "Channel Estimation and Performance Analysis of OneBit Massive MIMO Systems", IEEE Transactions on Signal Processing, Vol.65, No.15, pp.4075-4089, 2017.

[33] Y. Nan, L. Zhang, and X. Sun, "An Efficient DL Channel Estimation Approach for TDD Massive MIMO Systems", In: Proc. of IEEE Conf. on Vehicular Technology, pp.1-5, 2016.

[34] K. Guo, Y. Guo, G. Fodor, and G. Ascheid, "Uplink power control with MMSE receiver in multi-cell MU-massive-MIMO systems", In: Proc. of International Conf. on Communications, pp.5184-5190, 2014.

[35] R. F. Guiazon, K.K. Wong, and M. Fitch, "Coverage Probability of Cellular Networks Using Interference Alignment Under Imperfect CSI", Digital Communications and Networks, Vol.2, No.4, pp.162-166, 2016.

[36] V. H. M. Donald, "Advanced mobile phone service: The cellular concept", The Bell System Technical Journal, Vol.58, No.1, pp.15-41, 1979.

[37] D. Cox, "Cochannel Interference Considerations in Frequency Reuse SmallCoverage-Area Radio Systems", IEEE Transactions on Communications, Vol.30, No.1, pp.135-142, 1982.

[38] P. Rayi, M. V. S. Prasad, and P. V. V. Kishore, "Modelling, simulation of multi-user grouping considering channel states in MIMO systems", In: Proc. of International Conf. on Signal Processing and Communication Engineering Systems, pp. 231-235, 2015.

[39] Y. li, P. Fan, A. Leukhin, and L. Liu, "On the Spectral and Energy Efficiency of Full-Duplex Small Cell Wireless Systems with Massive MIMO", IEEE Transactions on Vehicular Technology, Vol.66, No.3, pp.2339-2353, 2017.

[40] P. Rayi, M. V. S. Prasad, and C. R. Reddy, "Capacity enhancement of DL massive MIMO systems with spatial channel correlation", In: Proc. of International Conf. on Inventive Computation Technologies, pp.1-6, 2016.

[41] P. Rayi and M. V. S. Prasad, "Enhancement of spectral and energy efficiency in massive MIMO systems with linear schemes", In: Proc. of International Conf. on Signal Processing, Communication and Networking, pp. 1-6, 2017. 